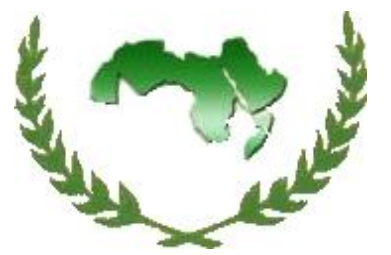

Arab Univ.

J. Agric. Sci.,

Ain Shams Univ.,

Cairo, 15(1), 131-139, 2007

\title{
EFFECT OF NITROGEN FERTIGATION LEVELS AND CHELATED CAL- CIUM FOLIAR APPLICATION ON THE PRODUCTIVITY OF SWEET CORN
}

\author{
Abou El-Yazied, A. ${ }^{1}$; M.E. Ragab'; Rawia, E. Ibrahim ${ }^{2}$ and S.M. Abou El-Wafa ${ }^{2}$ \\ 1- Horticulture Department, Faculty of Agriculture, Ain Shams University, Shoubra El- \\ Kheima, Cairo, Egypt \\ 2- Postharvest and Handling of Vegetable Department, Horticulture Research Institute, Agri- \\ cultural Research Center, Giza, Egypt
}

Keywords: Sweet corn, Zea mays, L. var. Saccharata, Nitrogen, Chelated calcium, Fertigation, Vegetative growth, Yield.

\begin{abstract}
This study was conducted in a sandy soil at Ali Mubarak Village Research Farm, South Tahrir Horticulture Research Station, Behaira Governorate, during the two successive seasons of 2002/2003 and 2003/2004 on sweet corn Jubilee hybrid. The study aimed to determine the best treatments of nitrogen fertigation level and chelated calcium foliar application on the productivity and quality of sweet corn. The obtained results indicated that increasing nitrogen fertigation level $(100,120,140$ to 160$) \mathrm{kg} \mathrm{N} / \mathrm{fed}$. and (or) chelated calcium $\left(12 \% \mathrm{Ca}^{++}\right)$concentration (500 and 1000 ppm Ca EDTA) led to increasing the vegetative growth (leaf area, plant height, fresh and dry weight), chemical composition (total chlorophyll, nitrogen, phosphorus, potassium and calcium in leaves) and yield characters (ear length, ear diameter, weight of 1000 seed, unhusked ear, husked ear and total yield) The promising treatment was 160 $\mathrm{kg}$ N/Fed. combined with 1000 ppm Ca EDTA under similar prevailing conditions of the present studying in a sandy soil.
\end{abstract}

\section{INTRODUCTION}

Sweet corn (Zea mays, L. var. Saccharata) is one of the most popular vegetables in many countries in the world. In Egypt, sweet corn is considered as an important crop for export in the future. Many investigators reported that sweet corn plant is highly sensitive to nitrogen fertilization during growth stages (Mullins et al 1999 and Nofal \&
Hinar, 2003). Darwish (2003) reported that increasing nitrogen fertilization led to increases in plant height, ear diameter, leaf area, $\mathrm{N}$ and protein in plant. Sanchez et al (1989); Salardini et al (1992) and Raja (2001) reported that increasing nitrogen fertilization increased significantly the green ear yield and kernel weight. Abd El-Megeid (2001) and Zebarth et al (2002) showed that increasing nitrogen fertilization caused significant differences in chlorophyll $\mathrm{A}$ and $\mathrm{B}$.

Calcium is essential for growth because it is needed for both cell division and cell elongation, Smith (1984) and Hunter et al (1995) stated that limes increased growth, yield, ear length, ear weight as well as the concentration of $\mathrm{N}, \mathrm{P}$ and $\mathrm{Ca}$ in sweet corn plants.

This study was therefore conducted to investigate the effect of nitrogen and chelated calcium on yield and quality of sweet corn.

\section{MATERIALS AND METHODS}

The present study was conducted in the Experimental Station of the Vegetable Research Department, at Ali Mubarak Village Research Farm, Behaira Governorate, during the period from 2002 to 2004, using sugary sweet corn hybrid, namely Jubilee. The soil analysis data indicate that the soil was sandy in texture, having pH 7.91 and 7.99, Ec. 1.51 and $1.49 \mathrm{ds} / \mathrm{m}$., organic matter 0.19 and 0.21 $\%$, contained 18 and $20 \mathrm{ppm}$ Nitrogen, 7 and 9 ppm Phosphorous, 10.5 and $10 \mathrm{ppm}$ Potassium and calcium content 20 and $22 \mathrm{ppm}$, in the first and second seasons, respectively. The field experiment was performed to investigate the response of sweet corn to nitrogen fertigation and foliar application of chelated calcium on vegetative characteristics, yield and its components. The

(Received April 2, 2006)

(Accepted January 22, 2007) 
seeds were sown directly in the soil on September 15 in both seasons. A split- plot system with three replicates was adopted where the studied nitrogen fertilization was situated in the main plots, and the foliar spray treatments were randomly distributed in the sub-plots. The plot area was $24 \mathrm{~m}^{2}$ consisted of 3 lines x $10 \mathrm{~m}$ length x $0.80 \mathrm{~m}$ width. Four nitrogen fertilization rates $(100,120,140$ and $160 \mathrm{~kg}$ nitrogen / fed.) were used in the form of ammonium sulphate $(20.5 \%)$ at four equal doses with drip irrigation system starting after 21days from sowing. Chelated calcium foliar applications were applied after 30 days from sowing (at the five-leaf stage) in eight sprays until maturity stage. Three levels of chelated calcium $\left(12 \% \mathrm{Ca}^{++}\right)$(Chelated calcium by EDTA produced by U.A.D. Co. Egypt) were studied: check (distilled water), 500 and 1000 ppm chelated calcium. The other Agricultural management "cultivation, manuring, fertigation, irrigation, pest and disease control were conducted, according to the recommendation of the Egyptian Ministry of Agriculture.

\section{Data recorded}

Vegetative growth measurements: Sixty plants were randomly taken at harvest time from each plot to record the leaf area (LA) per plant $\left(\mathrm{cm}^{2}\right)$, using the disk method, plant height $(\mathrm{cm})$ from the base to tassel top, plant fresh and dry weight (g).

Yield and ear parameters: Sixty ears from randomly plants of each plot were taken at harvest stage (milky stage) to determine ear length $(\mathrm{cm})$ (ear base to top) and diameter $(\mathrm{cm})$, number of rows/ear, number of kernels/row, un-husked and husked ear weight $(\mathrm{g})$, fresh and dry weight of 1000 kernels and total yield (g). All ears which were harvested from each plot were weighed to calculate the total yield.

Total chlorophyll was measured in fifth leaf by using Minolta chlorophyll meter model (SPAD502 instrument) from recently fully expended mature leaf. Total nitrogen, phosphorus, potassium and calcium were determined in dry fifth leaf. Total nitrogen was determined by Nesler method described in A.O.A.C. (1992). Phosphorus was colorimetrically determined as described by Jackson (1973) while potassium and calcium were measured by using flame photometer according to Brown and Lilliand (1946). Total nitrogen content was determined in kernels by using the modified micro kjeldhal apparatus of Parnus and Wagner and modified by Pregl (1945), then the ob- tained values were multiblied by 6.25 to calculate protein content.

Data obtained were statistically analyzed, according to Snedecor and Cochran (1989).

\section{RESULTS AND DISCUSSION}

\section{Vegetative growth}

Data presented in Table (1) indicated that in both seasons, increasing of the nitrogen applied levels increased the height, leaf area and fresh and dry weight of sweet corn plants. The addition of $160 \mathrm{~kg} \mathrm{~N} /$ fed gave the highest values of these growth parameters compared with the other studied levels. Similar results were obtained by Mullins et al (1999), Nofal \& Hinar (2003) and Grazia et al (2003) on sweet corn and maize. This favorable effect of $\mathrm{N}$ may be due its effective role in many biochemical processes within plants as it is necessary for cell protoplasm formation, photosynthesis activity in all plants and necessary for division and merestimic activity in plant organs, (Russel, 1973).

The data in the same Table showed that increasing of chelated calcium foliar application increased all tested plant growth characters. The applied calcium EDTA at 1000 ppm gave the most vigorous plants compared with the other foliar applications. These results were true in both seasons. These results may be attributed to the vital role of calcium inside plant tissues, as regulates plant activities, especially through protein phosphorylation, and it has major role in cell function and signal transduction (Poovaiah, 1986).

The obtained results agree with those of Kamel et al (1986); Salardini et al (1992); Hunter et al (1995); Padmaja et al (1999) and Hassan (2004).

As for the effect of interaction between nitrogen fertilization and chelated calcium foliar application, the application of $160 \mathrm{~kg} \mathrm{~N} / \mathrm{fed}$. with 1000 ppm calcium EDTA gave the highest value, while the lowest value was obtained from 100kg N/fed. without chelated calcium foliar application.

\section{Chemical composition}

\section{A- Total Chlorophyll}

Data presented in Table (2) indicated that applying nitrogen levels led to significant increase in the total chlorophyll content in both seasons. The addition of $160 \mathrm{~kg} \mathrm{~N} /$ fed gave the greatest total chlorophyll compared with the other levels. 
Table 1. Effect of nitrogen fertigation level and chelated calcium foliar application on vegetative growth of sweet corn plants in 2002/2003 and 2003/2004 seasons.

\begin{tabular}{|c|c|c|c|c|c|}
\hline $\begin{array}{c}\text { N level } \\
\text { ( kg/fed.) }\end{array}$ & $\begin{array}{c}\text { Ca-EDTA } \\
(\mathrm{ppm})\end{array}$ & $\begin{array}{l}\text { Plant height } \\
(\mathrm{cm})\end{array}$ & $\begin{array}{c}\text { Leaf area } \\
\left(\mathrm{cm}^{2}\right)\end{array}$ & $\begin{array}{c}\text { Fresh weight } \\
(\mathrm{g})\end{array}$ & $\begin{array}{c}\text { Dry weight } \\
(\mathrm{g})\end{array}$ \\
\hline \multicolumn{6}{|c|}{$2002 / 2003$ seasons } \\
\hline \multirow{3}{*}{100} & 0 & $163.32 \mathrm{j}$ & $313.58 \mathrm{k}$ & $261.20 \mathrm{j}$ & 98.711 \\
\hline & 500 & $164.85 \mathrm{i}$ & $318.58 \mathrm{j}$ & $265.85 \mathrm{i}$ & $103.71 \mathrm{k}$ \\
\hline & 1000 & $167.68 \mathrm{~h}$ & $322.55 \mathrm{i}$ & $271.24 \mathrm{~g}$ & $107.18 \mathrm{j}$ \\
\hline \multirow[t]{2}{*}{ Mean } & & 165.28 D & 318.24 D & 266.10D & 103.20D \\
\hline & 0 & $167.88 \mathrm{~g}$ & $322.92 \mathrm{~h}$ & $267.33 \mathrm{~h}$ & $108.58 \mathrm{i}$ \\
\hline \multirow[t]{2}{*}{120} & 500 & $168.58 \mathrm{f}$ & $323.48 \mathrm{~g}$ & $284.42 f$ & $109.40 \mathrm{~h}$ \\
\hline & 1000 & $169.80 \mathrm{e}$ & $328.49 \mathrm{e}$ & $292.54 \mathrm{e}$ & $113.29 \mathrm{~g}$ \\
\hline \multirow[t]{2}{*}{ Mean } & & 168.75 C & 324.97 C & 281.43C & $11042 C$ \\
\hline & 0 & $170.31 \mathrm{~d}$ & $325.24 \mathrm{f}$ & $323.09 \mathrm{~cd}$ & $116.56 f$ \\
\hline \multirow[t]{2}{*}{140} & 500 & $169.93 \mathrm{e}$ & $331.58 \mathrm{~d}$ & $327.44 b$ & $118.72 \mathrm{e}$ \\
\hline & 1000 & $170.24 \mathrm{~d}$ & $332.91 \mathrm{c}$ & $256.78 \mathrm{k}$ & $120.16 d$ \\
\hline \multirow[t]{2}{*}{ Mean } & & 170.16 B & 329.91 B & 302.44B & 118.48.B \\
\hline & 0 & $171.33 \mathrm{c}$ & $332.59 \mathrm{c}$ & $322.67 d$ & $121.38 \mathrm{c}$ \\
\hline \multirow[t]{2}{*}{160} & 500 & $173.93 \mathrm{~b}$ & $334.92 \mathrm{~b}$ & $323.88 \mathrm{c}$ & $125.38 b$ \\
\hline & 1000 & $175.58 \mathrm{a}$ & $335.91 \mathrm{a}$ & $365.52 \mathrm{a}$ & $128.38 \mathrm{a}$ \\
\hline \multirow[t]{2}{*}{ Mean } & & 173.62 A & 334.48 A & 334.35A & 125.05A \\
\hline & 0 & $168.21 \mathrm{C}$ & $323.58 \mathrm{C}$ & $293.57 \mathrm{C}$ & $111.31 \mathrm{C}$ \\
\hline \multirow[t]{4}{*}{ Average $\mathrm{Ca}$} & 500 & $169.32 \mathrm{~B}$ & $327.14 \mathrm{~B}$ & $300.40 \mathrm{~A}$ & 114.30B \\
\hline & 1000 & $170.82 \mathrm{~A}$ & $329.97 \mathrm{~A}$ & 296.52B & $117.25 \mathrm{~A}$ \\
\hline & & $2003 / 20$ & easons & & \\
\hline & 0 & $165.95 \mathrm{k}$ & $314.99 \mathrm{~h}$ & $260.55 j$ & $104.34 \mathrm{k}$ \\
\hline \multirow[t]{2}{*}{100} & 500 & $166.10 \mathrm{k}$ & $319.39 \mathrm{~g}$ & $265.90 \mathrm{k}$ & $108.24 j$ \\
\hline & 1000 & $167.29 \mathrm{j}$ & $323.91 \mathrm{f}$ & $270.94 \mathrm{i}$ & $110.23 \mathrm{i}$ \\
\hline \multirow[t]{2}{*}{ Mean } & & 166.45 D & 318.24 D & 265.80D & 17.60D \\
\hline & 0 & $167.88 \mathrm{~h}$ & $323.25 \mathrm{f}$ & $276.75 \mathrm{~h}$ & $110.24 \mathrm{i}$ \\
\hline \multirow[t]{2}{*}{120} & 500 & $167.62 \mathrm{i}$ & $325.51 \mathrm{e}$ & $283.35 \mathrm{~g}$ & $112.64 \mathrm{~h}$ \\
\hline & 1000 & $168.08 \mathrm{~g}$ & $329.38 \mathrm{~d}$ & $290.04 f$ & $118.58 \mathrm{f}$ \\
\hline \multirow[t]{2}{*}{ Mean } & & 167.86 C & 324.97 C & 283.38C & 113.82C \\
\hline & 0 & $169.28 \mathrm{e}$ & $329.99 \mathrm{~d}$ & $321.75 \mathrm{e}$ & $117.96 \mathrm{~g}$ \\
\hline \multirow[t]{2}{*}{140} & 500 & $168.98 \mathrm{f}$ & $331.98 \mathrm{c}$ & $325.76 c$ & $120.24 \mathrm{e}$ \\
\hline & 1000 & $169.75 \mathrm{~d}$ & $335.93 \mathrm{~b}$ & $353.00 \mathrm{i}$ & $121.43 \mathrm{~d}$ \\
\hline \multirow[t]{2}{*}{ Mean } & & 169.34 B & 329.91 B & 333.50B & $119.88 B$ \\
\hline & 0 & $172.71 \mathrm{c}$ & $332.34 \mathrm{c}$ & $324.67 d$ & $122.41 \mathrm{c}$ \\
\hline \multirow[t]{2}{*}{160} & 500 & $173.62 \mathrm{~b}$ & $336.39 \mathrm{~b}$ & $329.22 b$ & $126.95 b$ \\
\hline & 1000 & $174.41 \mathrm{a}$ & $337.97 \mathrm{a}$ & $365.04 \mathrm{a}$ & $128.93 a$ \\
\hline \multirow[t]{2}{*}{ Mean } & & $173.58 \mathrm{~A}$ & $334.48 \mathrm{~A}$ & $339.64 A$ & $126.10 \mathrm{~A}$ \\
\hline & 0 & $168.96 \mathrm{C}$ & $323.58 \mathrm{C}$ & $295.93 \mathrm{C}$ & $113.41 \mathrm{C}$ \\
\hline \multirow[t]{2}{*}{ average $\mathrm{Ca}$} & 500 & $169.08 \mathrm{~B}$ & 327.14 B & $301.06 \mathrm{~B}$ & $117.02 \mathrm{~B}$ \\
\hline & 1000 & $169.88 \mathrm{~A}$ & $329.97 \mathrm{~A}$ & $319.76 \mathrm{~A}$ & $119.79 \mathrm{~A}$ \\
\hline
\end{tabular}

*Any values within the column followed by the same latter are not statistically different; at the 0.05 level (Duncan's multiple range), small letters (interaction) 
Table 2. Effect of nitrogen fertigation level and chelated calcium foliar application on chemical contents of sweet corn leaves in 2002/2003 and 2003/2004 seasons.

\begin{tabular}{|c|c|c|c|c|c|c|}
\hline $\begin{array}{c}\text { N level } \\
\text { ( kg/fed.) }\end{array}$ & $\begin{array}{c}\text { Ca-EDTA } \\
(\mathrm{ppm})\end{array}$ & $\begin{array}{l}\text { Total chloro- } \\
\text { phyll (SPAD) }\end{array}$ & $\begin{array}{c}\text { Nitrogen } \\
\text { percentage }\end{array}$ & $\begin{array}{l}\text { Phosphorus } \\
\text { percentage }\end{array}$ & $\begin{array}{l}\text { Potassium } \\
\text { percentage }\end{array}$ & $\begin{array}{l}\text { Calcium } \\
\text { percentage }\end{array}$ \\
\hline \multicolumn{7}{|c|}{$2002 / 2003$ seasons } \\
\hline \multirow{3}{*}{100} & 0 & $44.38 \mathrm{f}$ & $1.41 \mathrm{f}$ & $0.452 \mathrm{i}$ & $1.02 \mathrm{~d}$ & $0.50 \mathrm{e}$ \\
\hline & 500 & $43.86 \mathrm{~g}$ & $1.41 \mathrm{f}$ & $0.458 \mathrm{~g}$ & $1.06 \mathrm{~cd}$ & $0.61 \mathrm{a}-\mathrm{e}$ \\
\hline & 1000 & $44.80 \mathrm{e}$ & $2.06 \mathrm{c}$ & $0.475 d$ & $1.11 \mathrm{~b}-\mathrm{c}$ & $0.65 \mathrm{a}-\mathrm{d}$ \\
\hline \multirow[t]{2}{*}{ Mean } & & 44.35 D & $1.62 \mathrm{D}$ & $0.475 \mathrm{D}$ & $1.06 \mathrm{C}$ & $0.59 \mathrm{C}$ \\
\hline & 0 & $46.65 \mathrm{~d}$ & $1.80 \mathrm{e}$ & $0.455 \mathrm{~h}$ & $1.09 \mathrm{~cd}$ & $0.53 \mathrm{de}$ \\
\hline \multirow[t]{2}{*}{120} & 500 & $46.31 \mathrm{~d}$ & $1.92 \mathrm{~d}$ & $0.462 f$ & $1.12 \mathrm{~b}-\mathrm{d}$ & $066 \mathrm{a}-\mathrm{d}$ \\
\hline & 1000 & $47.26 \mathrm{c}$ & $2.11 \mathrm{c}$ & $0.478 \mathrm{c}$ & $1.16 \mathrm{a}-\mathrm{d}$ & $0.68 \mathrm{a}-\mathrm{c}$ \\
\hline \multirow[t]{2}{*}{ Mean } & & 46.74 C & $1.95 \mathrm{C}$ & $0.465 C$ & $1.12 B C$ & $0.62 \mathrm{BC}$ \\
\hline & 0 & $49.49 \mathrm{a}$ & $1.91 \mathrm{~d}$ & $0.458 \mathrm{~g}$ & $1.14 \mathrm{a}-\mathrm{d}$ & $0.56 \mathrm{c}-\mathrm{e}$ \\
\hline \multirow[t]{2}{*}{140} & 500 & $47.61 \mathrm{c}$ & $2.09 \mathrm{c}$ & $0.463 f$ & $1.15 \mathrm{a}-\mathrm{d}$ & $0.69 \mathrm{a}-\mathrm{c}$ \\
\hline & 1000 & $47.38 \mathrm{c}$ & $2.21 \mathrm{~b}$ & $0.484 b$ & $1.18 \mathrm{a}-\mathrm{c}$ & $0.71 \mathrm{ab}$ \\
\hline \multirow[t]{2}{*}{ Mean } & & $48.49 \mathrm{~B}$ & 2.07 B & $0.468 B$ & $1.16 \mathrm{~B}$ & $0.65 \mathrm{AB}$ \\
\hline & 0 & $49.22 \mathrm{a}$ & $2.10 \mathrm{c}$ & $0.469 \mathrm{e}$ & $1.20 \mathrm{a}-\mathrm{c}$ & $0.58 \mathrm{~b}-\mathrm{e}$ \\
\hline \multirow[t]{2}{*}{160} & 500 & $48.69 \mathrm{~b}$ & $2.25 \mathrm{~b}$ & $0.474 d$ & $1.24 \mathrm{ab}$ & $0.70 \mathrm{a}-\mathrm{c}$ \\
\hline & 1000 & $49.25 \mathrm{a}$ & $2.31 \mathrm{a}$ & $0.492 \mathrm{a}$ & $1.28 \mathrm{a}$ & $0.73 \mathrm{a}$ \\
\hline Mean & & 49.05 A & $2.22 \mathrm{~A}$ & $0.478 \mathrm{~A}$ & $1.24 \mathrm{~A}$ & $0.67 \mathrm{~A}$ \\
\hline \multirow{3}{*}{$\begin{array}{c}\text { Average } \\
\mathrm{Ca}\end{array}$} & 0 & $47.43 \mathrm{~A}$ & $1.81 \mathrm{C}$ & $0.459 \mathrm{C}$ & $1.11 \mathrm{~A}$ & $0.54 \mathrm{C}$ \\
\hline & 500 & $46.62 \mathrm{~B}$ & $1.92 \mathrm{~B}$ & $0.464 \mathrm{~B}$ & $1.14 \mathrm{~A}$ & $0.66 \mathrm{~B}$ \\
\hline & 1000 & $47.42 \mathrm{~A}$ & $2.18 \mathrm{~A}$ & $0.482 \mathrm{~A}$ & $1.18 \mathrm{~A}$ & $0.69 \mathrm{~A}$ \\
\hline \multicolumn{7}{|c|}{ 2003/2004 seasons } \\
\hline \multirow{3}{*}{100} & 0 & $42.78 \mathrm{~h}$ & $1.70 \mathrm{j}$ & $0.450 \mathrm{~h}$ & $1.06 \mathrm{e}$ & $0.51 \mathrm{e}$ \\
\hline & 500 & $43.71 \mathrm{~g}$ & $1.91 \mathrm{~h}$ & $0.452 \mathrm{~g}$ & $1.09 \mathrm{de}$ & $0.62 \mathrm{~b}-\mathrm{d}$ \\
\hline & 1000 & $44.43 \mathrm{f}$ & $2.05 \mathrm{f}$ & $0.471 d$ & $1.12 \mathrm{c}-\mathrm{e}$ & $0.66 \mathrm{a}-\mathrm{c}$ \\
\hline \multirow[t]{2}{*}{ Mean } & & 43.64 D & $1.88 \mathrm{C}$ & $0.458 D$ & $1.09 \mathrm{C}$ & $0.60 \mathrm{~B}$ \\
\hline & 0 & $46.26 \mathrm{~d}$ & $1.78 \mathrm{i}$ & $0.458 \mathrm{f}$ & $1.09 \mathrm{de}$ & $0.54 \mathrm{de}$ \\
\hline \multirow[t]{2}{*}{120} & 500 & $45.85 \mathrm{e}$ & $1.95 \mathrm{~g}$ & $0.457 f$ & $1.14 \mathrm{~b}-\mathrm{e}$ & $0.67 \mathrm{a}-\mathrm{c}$ \\
\hline & 1000 & $46.48 \mathrm{~d}$ & $2.15 \mathrm{e}$ & $0.475 \mathrm{c}$ & $1.17 \mathrm{a}-\mathrm{d}$ & $0.69 \mathrm{a}-\mathrm{c}$ \\
\hline \multirow[t]{2}{*}{ Mean } & & 46.20 C & $1.96 \mathrm{~A}$ & $0.463 \mathrm{C}$ & 1.13BC & 0.63 B \\
\hline & 0 & $47.90 \mathrm{~b}$ & $1.21 \mathrm{k}$ & $0.458 \mathrm{f}$ & $1.15 \mathrm{~b}-\mathrm{d}$ & $0.59 \mathrm{c}-\mathrm{e}$ \\
\hline \multirow[t]{2}{*}{140} & 500 & $46.63 \mathrm{~d}$ & $2.18 \mathrm{~d}$ & $0.461 \mathrm{e}$ & $1.16 \mathrm{a}-\mathrm{d}$ & $0.70 \mathrm{a}-\mathrm{c}$ \\
\hline & 1000 & $47.53 \mathrm{bc}$ & $2.27 \mathrm{c}$ & $0.481 b$ & $1.19 \mathrm{a}-\mathrm{c}$ & $0.72 \mathrm{ab}$ \\
\hline \multirow[t]{2}{*}{ Mean } & & 47.35 B & $1.89 \mathrm{~B}$ & $0.467 B$ & 1.17B & $0.67 \mathrm{~A}$ \\
\hline & 0 & $48.32 \mathrm{a}$ & 1.191 & $0.461 \mathrm{e}$ & $1.19 \mathrm{a}-\mathrm{c}$ & $0.62 \mathrm{~b}-\mathrm{d}$ \\
\hline \multirow[t]{2}{*}{160} & 500 & $47.38 \mathrm{c}$ & $2.32 \mathrm{~b}$ & $0.471 \mathrm{~d}$ & $1.22 \mathrm{ab}$ & $0.72 \mathrm{ab}$ \\
\hline & 1000 & $48.33 \mathrm{a}$ & $2.37 \mathrm{a}$ & $0.486 a$ & $1.24 \mathrm{a}$ & $0.74 \mathrm{a}$ \\
\hline \multirow[t]{2}{*}{ Mean } & & $48.01 \mathrm{~A}$ & $1.96 \mathrm{~A}$ & $0.473 A$ & $1.22 \mathrm{~A}$ & $0.69 \mathrm{~A}$ \\
\hline & 0 & $46.32 \mathrm{~B}$ & $1.47 \mathrm{C}$ & $0.457 \mathrm{C}$ & $1.12 \mathrm{~A}$ & $0.56 \mathrm{C}$ \\
\hline \multirow{2}{*}{$\begin{array}{c}\text { average } \\
\mathrm{Ca}\end{array}$} & 500 & $45.89 \mathrm{C}$ & $2.09 \mathrm{~B}$ & $0.460 \mathrm{~B}$ & $1.15 \mathrm{~A}$ & $0.68 \mathrm{~B}$ \\
\hline & 1000 & $46.70 \mathrm{~A}$ & $2.21 \mathrm{~A}$ & $0.478 \mathrm{~A}$ & $1.18 \mathrm{~A}$ & $0.70 \mathrm{~A}$ \\
\hline
\end{tabular}

*Any values within the column followed by the same latter are not statistically different; at the 0.05 level (Duncan's multiple range), small letters (interaction). 
Table 3. Effect of nitrogen fertigation level and chelated calcium foliar application on ear characters of sweet corn in 2002/2003 and 2003/2004 seasons.

\begin{tabular}{|c|c|c|c|c|c|c|}
\hline $\begin{array}{c}\text { N level } \\
\text { ( kg/fed.) }\end{array}$ & $\begin{array}{c}\text { Ca-EDTA } \\
(\mathrm{ppm})\end{array}$ & $\begin{array}{l}\text { Total chloro- } \\
\text { phyll (SPAD) }\end{array}$ & $\begin{array}{c}\text { Nitrogen } \\
\text { percentage }\end{array}$ & $\begin{array}{l}\text { Phosphorus } \\
\text { percentage }\end{array}$ & $\begin{array}{l}\text { Potassium } \\
\text { percentage }\end{array}$ & $\begin{array}{c}\text { Calcium } \\
\text { percentage }\end{array}$ \\
\hline \multicolumn{7}{|c|}{$2002 / 2003$ seasons } \\
\hline \multirow{3}{*}{100} & 0 & $44.38 \mathrm{f}$ & $1.41 \mathrm{f}$ & $0.452 \mathrm{i}$ & $1.02 \mathrm{~d}$ & $0.50 \mathrm{e}$ \\
\hline & 500 & $43.86 \mathrm{~g}$ & $1.41 \mathrm{f}$ & $0.458 \mathrm{~g}$ & $1.06 \mathrm{~cd}$ & 0.61 a-e \\
\hline & 1000 & $44.80 \mathrm{e}$ & $2.06 \mathrm{c}$ & $0.475 d$ & $1.11 \mathrm{~b}-\mathrm{c}$ & $0.65 \mathrm{a}-\mathrm{d}$ \\
\hline \multirow[t]{2}{*}{ Mean } & & 44.35 D & $1.62 \mathrm{D}$ & $0.475 D$ & $1.06 \mathrm{C}$ & $0.59 \mathrm{C}$ \\
\hline & 0 & $46.65 \mathrm{~d}$ & $1.80 \mathrm{e}$ & $0.455 \mathrm{~h}$ & $1.09 \mathrm{~cd}$ & $0.53 \mathrm{de}$ \\
\hline \multirow[t]{2}{*}{120} & 500 & $46.31 \mathrm{~d}$ & $1.92 \mathrm{~d}$ & $0.462 \mathrm{f}$ & $1.12 b-d$ & 066 a-d \\
\hline & 1000 & $47.26 \mathrm{c}$ & $2.11 \mathrm{c}$ & $0.478 \mathrm{c}$ & $1.16 \mathrm{a}-\mathrm{d}$ & $0.68 \mathrm{a}-\mathrm{c}$ \\
\hline \multirow[t]{2}{*}{ Mean } & & 46.74 C & $1.95 \mathrm{C}$ & $0.465 C$ & $1.12 B C$ & $0.62 \mathrm{BC}$ \\
\hline & 0 & $49.49 \mathrm{a}$ & $1.91 \mathrm{~d}$ & $0.458 \mathrm{~g}$ & $1.14 \mathrm{a}-\mathrm{d}$ & $0.56 \mathrm{c}-\mathrm{e}$ \\
\hline \multirow[t]{2}{*}{140} & 500 & $47.61 \mathrm{c}$ & $2.09 \mathrm{c}$ & $0.463 \mathrm{f}$ & $1.15 \mathrm{a}-\mathrm{d}$ & $0.69 \mathrm{a}-\mathrm{c}$ \\
\hline & 1000 & $47.38 \mathrm{c}$ & $2.21 \mathrm{~b}$ & $0.484 b$ & $1.18 \mathrm{a}-\mathrm{c}$ & $0.71 \mathrm{ab}$ \\
\hline \multirow[t]{2}{*}{ Mean } & & 48.49 B & $2.07 \mathrm{~B}$ & $0.468 B$ & $1.16 B$ & $0.65 \mathrm{AB}$ \\
\hline & 0 & $49.22 \mathrm{a}$ & $2.10 \mathrm{c}$ & $0.469 \mathrm{e}$ & $1.20 \mathrm{a}-\mathrm{c}$ & $0.58 \mathrm{~b}-\mathrm{e}$ \\
\hline \multirow[t]{2}{*}{160} & 500 & $48.69 \mathrm{~b}$ & $2.25 \mathrm{~b}$ & $0.474 d$ & $1.24 \mathrm{ab}$ & $0.70 \mathrm{a}-\mathrm{c}$ \\
\hline & 1000 & $49.25 \mathrm{a}$ & $2.31 \mathrm{a}$ & $0.492 \mathrm{a}$ & $1.28 \mathrm{a}$ & $0.73 \mathrm{a}$ \\
\hline Mean & & 49.05 A & $2.22 \mathrm{~A}$ & $0.478 \mathrm{~A}$ & $1.24 \mathrm{~A}$ & $0.67 \mathrm{~A}$ \\
\hline \multirow{3}{*}{$\begin{array}{c}\text { Average } \\
\mathrm{Ca}\end{array}$} & 0 & $47.43 \mathrm{~A}$ & $1.81 \mathrm{C}$ & $0.459 \mathrm{C}$ & $1.11 \mathrm{~A}$ & $0.54 \mathrm{C}$ \\
\hline & 500 & $46.62 \mathrm{~B}$ & $1.92 \mathrm{~B}$ & $0.464 \mathrm{~B}$ & $1.14 \mathrm{~A}$ & $0.66 \mathrm{~B}$ \\
\hline & 1000 & $47.42 \mathrm{~A}$ & $2.18 \mathrm{~A}$ & $0.482 \mathrm{~A}$ & $1.18 \mathrm{~A}$ & $0.69 \mathrm{~A}$ \\
\hline \multicolumn{7}{|c|}{ 2003/2004 seasons } \\
\hline \multirow{3}{*}{100} & 0 & $42.78 \mathrm{~h}$ & $1.70 \mathrm{j}$ & $0.450 \mathrm{~h}$ & $1.06 \mathrm{e}$ & $0.51 \mathrm{e}$ \\
\hline & 500 & $43.71 \mathrm{~g}$ & $1.91 \mathrm{~h}$ & $0.452 \mathrm{~g}$ & $1.09 \mathrm{de}$ & $0.62 \mathrm{~b}-\mathrm{d}$ \\
\hline & 1000 & $44.43 \mathrm{f}$ & $2.05 \mathrm{f}$ & $0.471 \mathrm{~d}$ & $1.12 \mathrm{c}-\mathrm{e}$ & $0.66 \mathrm{a}-\mathrm{c}$ \\
\hline \multirow[t]{2}{*}{ Mean } & & 43.64 D & $1.88 \mathrm{C}$ & $0.458 D$ & $1.09 \mathrm{C}$ & 0.60 B \\
\hline & 0 & $46.26 \mathrm{~d}$ & $1.78 \mathrm{i}$ & $0.458 \mathrm{f}$ & $1.09 \mathrm{de}$ & $0.54 \mathrm{de}$ \\
\hline \multirow[t]{2}{*}{120} & 500 & $45.85 \mathrm{e}$ & $1.95 \mathrm{~g}$ & $0.457 f$ & $1.14 \mathrm{~b}-\mathrm{e}$ & $0.67 \mathrm{a}-\mathrm{c}$ \\
\hline & 1000 & $46.48 \mathrm{~d}$ & $2.15 \mathrm{e}$ & $0.475 \mathrm{c}$ & $1.17 \mathrm{a}-\mathrm{d}$ & $0.69 \mathrm{a}-\mathrm{c}$ \\
\hline \multirow[t]{2}{*}{ Mean } & & 46.20 C & $1.96 \mathrm{~A}$ & $0.463 \mathrm{C}$ & 1.13BC & 0.63 B \\
\hline & 0 & $47.90 \mathrm{~b}$ & $1.21 \mathrm{k}$ & $0.458 \mathrm{f}$ & $1.15 b-d$ & $0.59 \mathrm{c}-\mathrm{e}$ \\
\hline \multirow[t]{2}{*}{140} & 500 & $46.63 \mathrm{~d}$ & $2.18 \mathrm{~d}$ & $0.461 \mathrm{e}$ & $1.16 \mathrm{a}-\mathrm{d}$ & $0.70 \mathrm{a}-\mathrm{c}$ \\
\hline & 1000 & $47.53 \mathrm{bc}$ & $2.27 \mathrm{c}$ & $0.481 b$ & $1.19 \mathrm{a}-\mathrm{c}$ & $0.72 \mathrm{ab}$ \\
\hline \multirow[t]{2}{*}{ Mean } & & 47.35 B & $1.89 \mathrm{~B}$ & $0.467 B$ & 1.17B & 0.67 A \\
\hline & 0 & $48.32 \mathrm{a}$ & 1.191 & $0.461 \mathrm{e}$ & $1.19 \mathrm{a}-\mathrm{c}$ & $0.62 \mathrm{~b}-\mathrm{d}$ \\
\hline \multirow[t]{2}{*}{160} & 500 & $47.38 \mathrm{c}$ & $2.32 \mathrm{~b}$ & $0.471 \mathrm{~d}$ & $1.22 \mathrm{ab}$ & $0.72 \mathrm{ab}$ \\
\hline & 1000 & $48.33 \mathrm{a}$ & $2.37 \mathrm{a}$ & $0.486 a$ & $1.24 \mathrm{a}$ & $0.74 \mathrm{a}$ \\
\hline Mean & & 48.01 A & $1.96 \mathrm{~A}$ & $0.473 A$ & $1.22 \mathrm{~A}$ & 0.69 A \\
\hline \multirow{3}{*}{$\begin{array}{l}\text { average } \\
\mathrm{Ca}\end{array}$} & 0 & $46.32 \mathrm{~B}$ & $1.47 \mathrm{C}$ & $0.457 \mathrm{C}$ & $1.12 \mathrm{~A}$ & $0.56 \mathrm{C}$ \\
\hline & 500 & $45.89 \mathrm{C}$ & $2.09 \mathrm{~B}$ & $0.460 \mathrm{~B}$ & $1.15 \mathrm{~A}$ & $0.68 \mathrm{~B}$ \\
\hline & 1000 & $46.70 \mathrm{~A}$ & $2.21 \mathrm{~A}$ & $0.478 \mathrm{~A}$ & $1.18 \mathrm{~A}$ & $0.70 \mathrm{~A}$ \\
\hline
\end{tabular}

*Any values within the column followed by the same latter are not statistically different; at the 0.05 level (Duncan's multiple range), small letters (interaction). 
Table 4. Effect of nitrogen fertigation level and chelated calcium foliar application on yield characters of sweet corn plants in 2002/2003 and 2003/2004 seasons.

\begin{tabular}{|c|c|c|c|c|c|}
\hline $\begin{array}{l}\text { N level } \\
(\mathrm{kg} / \mathrm{fed} .)\end{array}$ & $\begin{array}{c}\text { Ca-EDTA } \\
(\mathrm{ppm})\end{array}$ & $\begin{array}{l}\text { Ear length } \\
(\mathrm{cm})\end{array}$ & $\begin{array}{c}\text { Ear diameter } \\
(\mathrm{cm})\end{array}$ & $\begin{array}{c}\text { Number of } \\
\text { rows/ear }\end{array}$ & $\begin{array}{l}\text { Number of } \\
\text { kernels/row }\end{array}$ \\
\hline \multicolumn{6}{|c|}{$2002 / 2003$ seasons } \\
\hline \multirow{3}{*}{100} & 0 & $18.19 \mathrm{e}$ & $3.89 \mathrm{i}$ & 33.931 & $16.28 \mathrm{f}$ \\
\hline & 500 & $18.49 \mathrm{~d}$ & $3.99 \mathrm{~h}$ & $34.76 \mathrm{i}$ & $16.32 \mathrm{ef}$ \\
\hline & 1000 & $18.76 \mathrm{c}$ & $4.19 \mathrm{~g}$ & $34.54 \mathrm{j}$ & $16.44 d$ \\
\hline \multirow[t]{2}{*}{ Mean } & & 18.48 D & 4.02 D & 34.41D & 16.35D \\
\hline & 0 & $18.25 \mathrm{e}$ & $4.21 \mathrm{f}$ & $34.33 \mathrm{k}$ & $16.35 \mathrm{e}$ \\
\hline \multirow[t]{2}{*}{120} & 500 & $18.71 \mathrm{c}$ & $4.24 \mathrm{e}$ & $35.72 \mathrm{~g}$ & $16.48 \mathrm{~cd}$ \\
\hline & 1000 & $19.10 \mathrm{~b}$ & $4.20 \mathrm{fg}$ & $35.60 \mathrm{~h}$ & $16.49 \mathrm{~cd}$ \\
\hline \multirow[t]{2}{*}{ Mean } & & $18.69 \mathrm{C}$ & $4.22 \mathrm{C}$ & $35.21 \mathrm{C}$ & $16.44 \mathrm{C}$ \\
\hline & 0 & $18.69 \mathrm{c}$ & $4.23 \mathrm{e}$ & $36.07 f$ & $16.42 \mathrm{~d}$ \\
\hline \multirow[t]{2}{*}{140} & 500 & $19.45 \mathrm{a}$ & $4.32 \mathrm{~d}$ & $36.91 b$ & $16.52 b c$ \\
\hline & 1000 & $19.45 \mathrm{a}$ & $4.34 \mathrm{c}$ & $36.42 \mathrm{e}$ & $16.58 b$ \\
\hline \multirow[t]{2}{*}{ Mean } & & 19.20 B & $4.30 \mathrm{~B}$ & 36.47B & $16.50 \mathrm{~B}$ \\
\hline & 0 & $19.11 b$ & $4.24 \mathrm{e}$ & $36.67 d$ & 16.44 \\
\hline \multirow[t]{2}{*}{160} & 500 & $19.19 \mathrm{~b}$ & $4.43 \mathrm{a}$ & $37.06 \mathrm{a}$ & $16.74 \mathrm{a}$ \\
\hline & 1000 & $19.49 \mathrm{a}$ & $4.37 \mathrm{~b}$ & $36.82 \mathrm{c}$ & $16.68 \mathrm{a}$ \\
\hline \multirow[t]{2}{*}{ Mean } & & $19.26 \mathrm{~A}$ & $4.32 \mathrm{~A}$ & $36.85 \mathrm{~A}$ & $16.62 \mathrm{~A}$ \\
\hline & 0 & $18.56 \mathrm{C}$ & $4.14 \mathrm{C}$ & $35.25 \mathrm{C}$ & 16.37B \\
\hline \multirow[t]{4}{*}{ Average $\mathrm{Ca}$} & 500 & 18.96B & $4.22 \mathrm{~B}$ & $36.11 \mathrm{~A}$ & $16.51 \mathrm{~A}$ \\
\hline & 1000 & $19.20 \mathrm{~A}$ & $4.28 \mathrm{~A}$ & $35.84 \mathrm{~B}$ & $16.55 \mathrm{~A}$ \\
\hline & & $2003 / 2$ & seasons & & \\
\hline & 0 & $17.73 \mathrm{j}$ & $3.91 \mathrm{~g}$ & $34.24 \mathrm{k}$ & $16.05 \mathrm{~h}$ \\
\hline \multirow[t]{2}{*}{100} & 500 & $18.21 \mathrm{i}$ & $4.05 \mathrm{f}$ & $34.92 \mathrm{j}$ & $16.67 f$ \\
\hline & 1000 & $18.85 \mathrm{~g}$ & $4.17 \mathrm{e}$ & $36.67 \mathrm{~h}$ & 16.72ef \\
\hline \multirow[t]{2}{*}{ Mean } & & $18.26 \mathrm{D}$ & $4.04 \mathrm{D}$ & 35.28D & 16.48D \\
\hline & 0 & $18.67 \mathrm{~h}$ & $4.19 \mathrm{~d}$ & $35.74 \mathrm{i}$ & $16.51 \mathrm{~g}$ \\
\hline \multirow[t]{2}{*}{120} & 500 & $18.69 \mathrm{~h}$ & $4.24 \mathrm{c}$ & $36.89 \mathrm{~g}$ & $16.69 f$ \\
\hline & 1000 & $19.10 \mathrm{f}$ & $4.20 \mathrm{~d}$ & $37.31 \mathrm{f}$ & $16.77 \mathrm{de}$ \\
\hline \multirow[t]{2}{*}{ Mean } & & 18.82 C & $4.21 \mathrm{C}$ & $36.64 \mathrm{C}$ & $16.66 \mathrm{C}$ \\
\hline & 0 & $19.24 \mathrm{e}$ & $4.21 \mathrm{~d}$ & $38.08 \mathrm{e}$ & $16.67 f$ \\
\hline \multirow[t]{2}{*}{140} & 500 & $19.52 \mathrm{~d}$ & $4.20 \mathrm{~d}$ & $38.24 d$ & $17.06 \mathrm{c}$ \\
\hline & 1000 & $19.77 \mathrm{~b}$ & $4.35 \mathrm{a}$ & $36.64 \mathrm{~h}$ & $17.21 \mathrm{~b}$ \\
\hline \multirow[t]{2}{*}{ Mean } & & $19.51 \mathrm{~B}$ & $4.25 \mathrm{~B}$ & 37.65B & 16.98B \\
\hline & 0 & $19.66 \mathrm{c}$ & $4.24 \mathrm{c}$ & $38.41 \mathrm{c}$ & $16.81 d$ \\
\hline \multirow[t]{2}{*}{160} & 500 & $19.75 \mathrm{~b}$ & $4.31 \mathrm{~b}$ & $39.14 a$ & $17.52 \mathrm{a}$ \\
\hline & 1000 & $19.97 \mathrm{a}$ & $4.36 \mathrm{a}$ & $38.74 b$ & $17.52 \mathrm{a}$ \\
\hline \multirow[t]{2}{*}{ Mean } & & 19.79 A & $4.30 \mathrm{~A}$ & 38.77A & $17.28 \mathrm{~A}$ \\
\hline & 0 & $18.82 \mathrm{C}$ & $4.14 \mathrm{C}$ & $36.62 \mathrm{C}$ & $16.51 \mathrm{C}$ \\
\hline \multirow[t]{2}{*}{ average $\mathrm{Ca}$} & 500 & $19.04 \mathrm{~B}$ & $4.20 \mathrm{~B}$ & 37.30B & 16.99B \\
\hline & 1000 & $19.42 \mathrm{~A}$ & 4.37 A & $37.34 \mathrm{~A}$ & $17.05 \mathrm{~A}$ \\
\hline
\end{tabular}

*Any values within the column followed by the same latter are not statistically different; at the 0.05 level (Duncan's multiple range), small letters (interaction) 
Similar results are reported by Abd El-Megeid (2001) and Zebarth et al (2002).

Data indicated also that the spraying of chelated calcium at $1000 \mathrm{ppm}$ gave the greatest total chlorophyll compared with the other foliar applications. These results were true in both seasons.

The interaction between nitrogen fertilization and chelated calcium foliar application showed that applying $160 \mathrm{~kg} \mathrm{~N} / \mathrm{fed}$. combined with 1000 ppm chelated calcium gave the highest chlorophyll value, while the lowest values were obtained from $100 \mathrm{~kg} \mathrm{~N} / \mathrm{fed}$. without chelated calcium foliar application.

\section{B- Mineral content}

Data shown in Table (2) indicted that the applied nitrogen levels caused significant increment in percentage of nitrogen, phosphorus, potassium, calcium in the fifth leaf. The most effective level in increasing $\mathrm{N}, \mathrm{P}, \mathrm{K}$ and Ca contents was $160 \mathrm{~kg}$ N/ Fed. in both seasons. Similar results were obtained by Taber \& Cox (1983); Smith (1984); Thiraporn et al (1992) and Hassan (2004).

Concerning the effect of chelated calcium, data showed that chelated calcium applied at $1000 \mathrm{ppm}$ gave the highest percentage of $\mathrm{N}, \mathrm{P}$ and $\mathrm{Ca}$ in the fifth leaf, compared with the other foliar applications. These results were true in both seasons. The results agree with those obtained by Smith (1984). Whereas, data showed that foliar application of chelated calcium did not significantly affect potassium percentage, in the two tested seasons. The obtained results agree with those of Smith (1984) and Hunter et al (1995).

The interaction between nitrogen application levels and chelated calcium foliar sprays was significant in both seasons. The addition of $160 \mathrm{~kg}$ N/fed combined with 1000ppm chelated calcium showed the highest values of $\mathrm{N}, \mathrm{P}, \mathrm{K}$ and $\mathrm{Ca}$, while the lowest values of these nutrients were obtained from $100 \mathrm{~kg} \mathrm{~N} / \mathrm{fed}$. without chelated calcium addition in both seasons.

The satisfactory obtained effects on plant growth (Table 1) as a result of applying the highest level of $\mathrm{N}$ fertigation and $\mathrm{Ca}$ foliar application may be also attributed to the increasing the concentration of $\mathrm{N}, \mathrm{P}, \mathrm{K}$ and Ca chelated as well as chlorophyll in leaf tissues.

\section{Yield and its components}

\section{A- Ear characters}

Data shown in Table (3) indicated that each increase in the applied nitrogen level (100, 120,
140 and $160 \mathrm{~kg} \mathrm{~N} /$ Fed.) caused significant increase in the ear length,ear diameter, number of rows/ear and number of kernels/row in both seasons. These results may be due to the numerous effects of nitrogen fertilizer on plant growth. Several investigators reached to the same results Smith, 1984; Raja, 2001; Darwish, 2003; Oraby et al 2003 and Hassan, 2004.

The increasing of chelated calcium foliar application increased the ear length,ear diameter, number of rows/ear and number of kernels/row. The spraying of chelated chelated calcium at 1000 ppm gave the highest values compared with the other foliar applications. These results were true in both seasons. The results are agree with those obtained by Smith (1984) and Hunter et al (1995).

As to the effect of interaction between nitrogen fertilization and foliar application, the addition of $160 \mathrm{~kg} \mathrm{~N} / \mathrm{fed}$. combined with spraying with $1000 \mathrm{ppm}$ chelated calcium gave the highest values of ear length, ear diameter, number of rows/ear and number of kernels/row, while the lowest values were obtained from $100 \mathrm{~kg} \mathrm{~N} / \mathrm{fed}$. without chelated calcium foliar application.

\section{B- Yield characters}

Data presented in Table (4) indicated that each increase in the applied nitrogen level caused significant increase in weight of 1000 seed, unhusked ear, husked ear and yield of ears in both seasons. The application of $160 \mathrm{~kg} \mathrm{~N} / \mathrm{fed}$ gave the greatest ear yield compared with the other levels. Similar results are reported by Sanchez et al (1989); Salardini et al (1992); Stone et al (1998); Saied \& Gaber (1999); Raja (2001); Grazia et al (2003) and Oraby et al (2003).

As for chelated calcium effects, spraying with chelated calcium at $1000 \mathrm{ppm}$ produced the greatest weight of 1000 seed, unhusked ear, husked ear and ear yield compared with the other foliar application levels. These results were true in both seasons The results are in agreement with those obtained by Smith (1984) and Hunter et al (1995).

The interaction between nitrogen fertilization and foliar application was significant in both seasons. The application of $160 \mathrm{~kg} \mathrm{~N} / \mathrm{fed}$. combined with 1000ppm calcium EDTA resulted in the highest weight of 1000 seed, unhusked ear, husked ear and ear yield value, while the lowest value was obtained from $100 \mathrm{~kg}$ N/fed. without chelated calcium foliar application. The obtained increase in ear yield as well the improvement in ear characters with resulted from applying the highest levels 
of $\mathrm{N}$ fertigation and $\mathrm{Ca}$ foliar application may be attributed to their stimulating influences on plant growth (Table 1) as well as chemical composition (Table 2).

\section{REFERENCES}

Abd El-Megeid, M.A. (2001). Effect of Different NP Levels on the Chemical Composition of Maize Plant. P. 142. Ph.D. Thesis, Fac. Agric., Cairo Univ.

A.O.A.C. (1992). Official Methods of Analysis, of Agricultural Chemists $12^{\text {th }}$ Ed. Washington, D.C., USA.

Brown, J.D. and O. Lilliand (1946). Rapid determination of potassium and sodium in plant material and soil extracts by flame photometry, Proc. Amer. Soc. Hort. Sci., (48): 341-346.

Darwish, A.A. (2003). The yield and yield components of maize as influenced by nitrogen, zinc and boron fertilization. J. Agric. Sci., Mansoura Univ., 28(2): 799-810.

Grazia, J.D.; P.A. Tittonell; D. Germinara and A.D. Chiesa (2003). Phosphorus and nitrogen fertilization in sweet corn (Zea mays L.var. saccharata bailey). Spanish J. Agri. Res. 1(2):103107.

Hassan, M.M. (2004). Response Yellow Maize to Nitrogen Potassium Fertilization and Plant Density. P. 172. Ph. D. Thesis, Fac. Agric., AlAzhar Univ., Cairo, Egypt.

Hunter, D.J.; L.G. Yapa; N.V. Hue and M. Eaqub (1995). Comparative effects of green manure and lime on the growth of sweet corn and chemical properties of an acid Oxisol in Western Samoa Corn. Commu. Soil. Sci. and Plant Anal. 26 (3\&4): 375-388.

Jackson, M.L. (1973). Soil Chemical Analysis. Prentice- Hall of India Private. Limited. New Delhi.

Kamel, M.S.; S.A. Shaban; A.I. Ashour and E.M. Abdel-Lateef (1986). Soil and foliar fertilization studies on maize yield and yield components. Proc. $2^{\text {nd }}$ Conf. Agron., 1: 247-261.

Mullins, C.A.; R.A. Straw; B. J. Pitt; D.O. Onks; M.D. Mullen; J. Reynolds and M. Kirchner (1999). Response of selected sweet corn cultivars to nitrogen fertilization. HortTechnology 1 (9): 32-35.

Nofal, A. and A.S. Hinar (2003). Growth and chemical properties of maize grains of some single crosses as affect by nitrogen fertilization and manure under sprinkler irrigation in a sandy soil. Egypt. J. Appl. Sci., 18(58): 583-596.
Oraby, F.T.; A.A. Sarhan, M.F. AbdelMaksoud and A.H. Bassiouny (2003). Proper agronomic practices required to maximize productivity of some maize varieties in old and reclaimed soils. Effect of sowing dates on response of two maize hybrids to nitrogen fertilization. Egypt. J. Appl. Sci., 18: (58): 597-618.

Padmaja, M.; D. Sreelatha and K.L. Roa (1999). Effect of nitrogen on nutrient uptake in maize (Zea mays L.) Types. J. Res. Angrau., 27 (4): 112-114.

Poovaiah, B.W. (1986). Role of calcium in prolonging storage life of fruits and vegetable. Food Technol., 40: 86-89.

Pregl, F. (1945). Quantitive Organic Micro Analysis $4^{\text {th }}$ Ed. J. and A. Churchill LTD., London.

Raja, V. (2001). Effect of nitrogen and plant population on yield and quality of super sweet corn (Zea mays). Indian J. Agron., 46 (2): 264-269.

Russel, E.W. (1973). Soil Condition and Plant Growth. 10 ${ }^{\text {th }}$ Ed. Longman Group Limited, London.

Saied E.M. and E.M.E. Gaber (1999). Response of some maize varieties to nitrogen fertilization and plant density. J. Agric. Sci. Mansoura Univ., 24(4): 1665-1675.

Salardini, A.A.; L.A. Sparrow and R.J. Holloway (1992). Sweet corn responses to basal and top-dressed and sources of nitrogenous fertilizers. Aust. J. Agric. Res., 43 (1): 171-180.

Sanchez, C.A.; R.T. Nagata and V.L. Guzman (1989). Response of sweet corn to nitrogen source and rate on Histosols. HortScience 24 (6): 925927.

Smith, C.B. (1984). Sweet corn growth responses and leaf concentrations as affected by lime types and fertilizer treatments in a five- year study. $\mathbf{J}$. Amer. Soc. Hort. Sci., 109(4): 572-577.

Snedecor, G.W. and W.G. Cochran (1989). Statistical Methods. $8^{\text {th }}$ Ed. Iowa State Univ. Press, Ames, Iowa, USA.

Stone, P.J.; I.B. Sorensen and J.B. Reid (1998). Effect of plant population and nitrogen fertilizer on yield and quality of super sweet corn. Proc. Annual Conf. Agro. Soci. New. Zealand 28: 1-5. Taber, H.G. and D.F. Cox (1983). Nitrogen effect on yield and kernel protein content of sweet corn grown on sandy soils. Comm. Soil Sci. and Plant Anal. 14 (7): 585-599.

Thiraporn, R.; B. Feil and P. Stamp (1992). Effect of nitrogen fertilization on yield and accumulation of nitrogen, phosphorus and potassium in 
grains of tropical maize. J. Agron. Crop Sci., 169 (1-2): 9-16.

Zebarth, B.J.; M. Younie; J.W. Paul and S. Bittman (2002). Evaluation of leaf chlorophyll index for making fertilizer nitrogen recommendation for silage corn in high fertility environment.

Commun. Soil Sci. Plant. Anal. 33(5\&6): 665684.

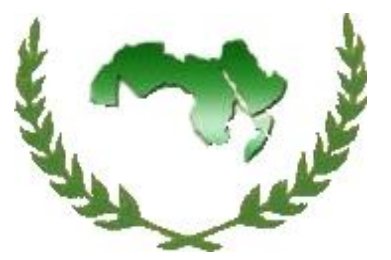

مجلة اتحاد الجامعات العربية للار اسات و البحوث الزعاد الزعات العزية

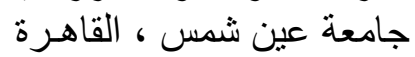

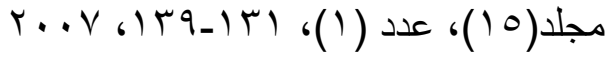

تأثير معدلات التسميد التيتروجيني مع ماء الري والرش الورقي بالكالسيوم المخلبي على إنتاجية

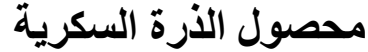

$[1 r]$

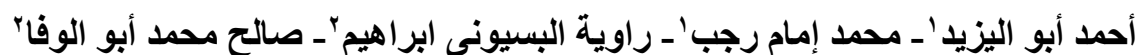

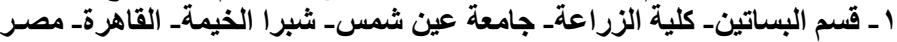

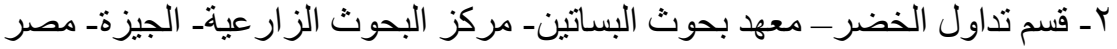

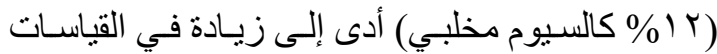

أجريت هذه الدراسـة بالمزر عـة البحثبة بقريـة على

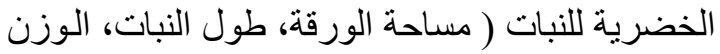

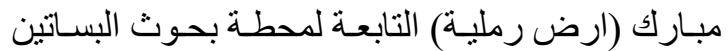

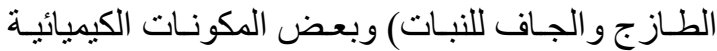

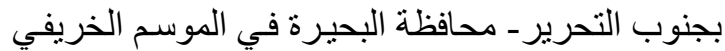

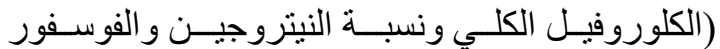

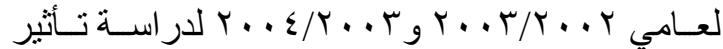

و البوتاسيو و الكالسيوم في الأوراق) وتحسين الصفات

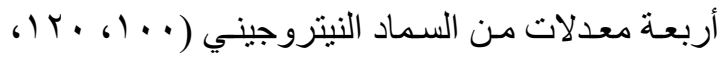

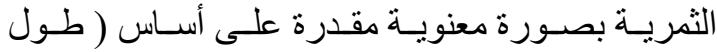

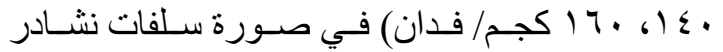

الكوز، قطر الكوز، المحصول الكلى).

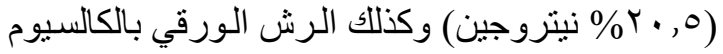

ويمكن الإستتناج بأن المعاملة بإستخدام • 17 ( كجم/ فدان الكول

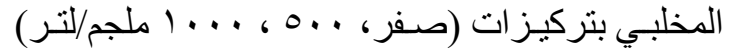

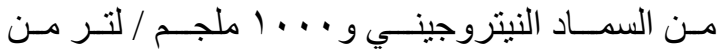

على محصول الذرة السكرية هجين جوبيلى. و أوضحت

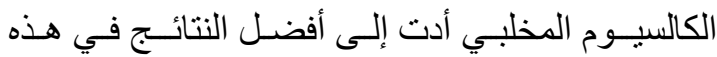

النتائج انها بزيادة مستوى التسميد بعنصر النيتروجين

الدر اسة تحت ظروف الأر اضي الرملية.

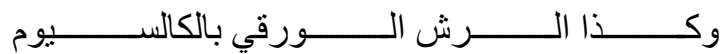

